\title{
Hotspot Update, and a new Excess of Events on the Sky Seen by the Telescope Array Experiment
}

\section{Jihyun Kim, ${ }^{a, *}$ Dmitri Ivanov, ${ }^{a}$ Kazumasa Kawata, ${ }^{b}$ Hiroyuki Sagawa ${ }^{b}$ and Gordon Thomson $^{a}$ on behalf of the Telescope Array Collaboration}

(a complete list of authors can be found at the end of the proceedings)

${ }^{a}$ High Energy Astrophysics Institute and Department of Physics and Astronomy, University of Utah Salt Lake City, Utah, USA

${ }^{b}$ Institute for Cosmic Ray Research, University of Tokyo

Kashiwa, Chiba, Japan

E-mail: jihyun@cosmic.utah.edu

The Telescope Array (TA) experiment, the largest observatory studying ultrahigh energy cosmic rays in the northern hemisphere, has reported an excess in the arrival direction distribution for events with energies above $5.7 \times 10^{19} \mathrm{eV}$, called the hotspot. We report here the latest results of the TA hotspot using the most recent data measured by the TA surface detector array, which is more than doubled exposure since the first publication; the hotspot still exists with 3 sigma post-trial significance. By using an oversampling search with a $20^{\circ}$-circle, similar to the study of the hotspot, we find an additional excess of events at slightly lower energies. The Perseus-Pisces supercluster lies at the location of the new excess. Assuming this structure is responsible for the excess, we conducted a statistical analysis to verify the correlation between observed events and the members of the Perseus-Pisces supercluster.

$37^{\text {th }}$ International Cosmic Ray Conference (ICRC 2021)

July 12 th - 23rd, 2021

Online - Berlin, Germany

\footnotetext{
*Presenter
} 


\section{Introduction}

Ultra-high-energy cosmic rays (UHECRs) are energetic charged particles originating from outer space that impinge on the earth's atmosphere with energies above $10^{18} \mathrm{eV}$. There have been many experiments to find out the nature and origin UHECRs for more than 60 years. Owing to these efforts, we have found some answers to the physics of UHECRs. First, the significant suppression of UHECR flux above $\sim 5 \times 10^{19} \mathrm{eV}$ has been confirmed [1-3]. In addition, the results of mass composition show that the primary particles of UHECRs would be protons or light nuclei or a mixed composition; heavy nuclei like iron is excluded [4-7]. However, the sources of UHECRs are still unknown as yet.

The first step toward finding out the sources of UHECRs is searching for anisotropy in the arrival directions of the UHECRs, particularly in relation to the inhomogeneous distribution of matter in the nearby universe in which the sources of the UHECRs reside. Recently, there have been reports about the anisotropy of the arrival direction of UHECRs, as detected by the Telescope Array (TA) experiment and the Pierre Auger Observatory, respectively [8, 9].

In 2014, the TA experiment reported a cluster of UHECR events with energy greater than $57 \mathrm{EeV}\left(1 \mathrm{EeV}=10^{18} \mathrm{eV}\right)$ in the northern sky, called the hotspot [10]. By using 5 years of data collected by the TA surface detector array, we showed oversampling analysis results using an intermediate angular scale of $20^{\circ}$. The significance map was obtained using the Li-Ma analysis by comparing the data to the isotropic background events. It was found that the maximum significance of $5.1 \sigma$ at the position of $\left(146.7^{\circ}, 43.2^{\circ}\right)$ in the equatorial coordinates, and the probability that such clustered events can be observed by chance in an isotropic UHECR sky, is estimated to be around $3.4 \sigma$ level by Monte-Carlo (MC) simulations. In this work, we update on the hotspot using the most recent data and report an additional excess of events at slightly lower energies in the region of the Perseus-Pisces supercluster.

\section{Telescope Array experiment}

The TA experiment is the largest cosmic ray observatory in the northern hemisphere, which is located in the west desert of Utah, USA $\left(39.3^{\circ} \mathrm{N}\right.$ and $112.9^{\circ} \mathrm{W}, 1400 \mathrm{~m}$ above sea level). It is a hybrid observatory, consisting of a surface detector (SD) array and three fluorescence detector (FD) stations, to observe the extensive air showers induced by UHECRs. The SD array consists of 507 plastic scintillation detectors deployed on a square grid with $1.2 \mathrm{~km}$ spacing, covering an area of approximately $700 \mathrm{~km}^{2}$ [11]. The FD stations, having 38 telescopes, overlook the area of the SD array [12].

\section{Datasets}

Here, we used two types of dataset measured by the TA SD array for each study: the hotspot and a new excess at slightly lower energies. To examine lower energy events, we used the data collected over a data-taking period of 11 years, from May 11 of 2008 to May 10 of 2019. The event selection criteria that apply to the data are as follows:

1. Energy $\geq 10^{19.4} \mathrm{eV}$, 
2. Zenith angle of arrival direction $<55^{\circ}$,

3. At least five SDs triggered,

4. Shower geometry and lateral distribution function fit $\chi^{2} /$ dof $<4$,

5. Reconstructed pointing direction error $<5^{\circ}$,

6. The fractional uncertainty in $\mathrm{S}(800)<25 \%$,

7. The largest signal counter surrounded by four working counters, there must be one working counter to the left, right, down, up on the grid of the largest signal counter, but they do not have to be immediate neighbors of the largest signal counter.

The energy of reconstructed events is determined by SD and renormalized by $1 / 1.27$ to match the FD energy scale determined calorimetrically [13]. There are 864 events with $E \geq 10^{19.4} \mathrm{eV}$ in the dataset after the selection. The energy and angular resolution of events range from $10 \%$ $(E \geq 57 \mathrm{EeV})$ to $20 \%\left(E \geq 10^{19.4} \mathrm{eV}\right)$ and from $1.0^{\circ}$ to $1.5^{\circ}$, respectively [14].

For the hotspot analysis, we used the data recorded for 12 years, from May 11 of 2008 to May 10 of 2020. We adopted the selection criteria used for the original hotspot study, which are looser than those for lower energy by eliminating the border cut used in the original analysis to remove events near the array boundaries, and the minimum number of triggered SD is four. Therefore, we could take advantage of more observed cosmic ray statistics, keeping reasonable energy and angular resolution for investigating intermediate scale cosmic ray anisotropy [8]. There are 179 events having energies greater than $57 \mathrm{EeV}$.

\section{Update on the hotspot}

To investigate an intermediate scale anisotropy, we conducted the oversampling analysis. First, we set $0.1^{\circ} \times 0.1^{\circ}$ grid in the equatorial coordinates. At each grid point, we summed over the number of events in a given angular scale circle for the data, defined as $N_{\text {on }}$. $\left(N_{\text {off }}=N_{\text {tot }}-N_{\text {on }}\right)$ Then, we generated $10^{5}$ events assuming an isotropic flux taking into account the geometrical exposure $g(\theta)=\sin \theta \cos \theta$ as a function of zenith angle $(\theta)$ because the detection efficiency for this energy range is $\sim 100 \%$ regardless of zenith angle $\theta$. Using these isotropic background events, we summed over the number of events in the given angular scale circle in the same manner we did for the data, that defines $\alpha=N_{\text {iso, on }} / N_{\text {iso, off }}$ for exposure ratio of on to off. Then, by comparing the oversampling of observed data to the isotropic background events, the statistical significance of the excess of events at each grid point is calculated by the following equation [15]:

$$
S_{\mathrm{LM}}=\sqrt{2}\left[N_{\text {on }} \ln \left(\frac{(1+\alpha) N_{\text {on }}}{\alpha\left(N_{\text {on }}+N_{\text {off }}\right)}\right)+N_{\text {off }} \ln \left(\frac{(1+\alpha) N_{\text {off }}}{N_{\text {on }}+N_{\text {off }}}\right)\right]^{1 / 2} .
$$

For the hotspot analysis, we used 179 events with energies greater than $57 \mathrm{EeV}$ recorded by TA SDs for 12 years, selected by the criteria described in the previous section. Five oversampling angular scales, $15^{\circ}, 20^{\circ}, 25^{\circ}, 30^{\circ}$, and $35^{\circ}$, were introduced, and the Li-Ma analysis was conducted at each grid point to examine anisotropy in arrival direction distribution. For each angular scale, we search for the maximum Li-Ma significance and its positions. Among them, when we used a $25^{\circ}$ circle for oversampling, the most significant excess to the isotropy was found at $\left(144.0^{\circ}, 40.5^{\circ}\right)$ in the equatorial coordinates. Inside the $25^{\circ}$-circle from the position, 40 out of 179 events are detected, whereas only 14.6 events are expected from the isotropic distribution. The Li-Ma significance is 

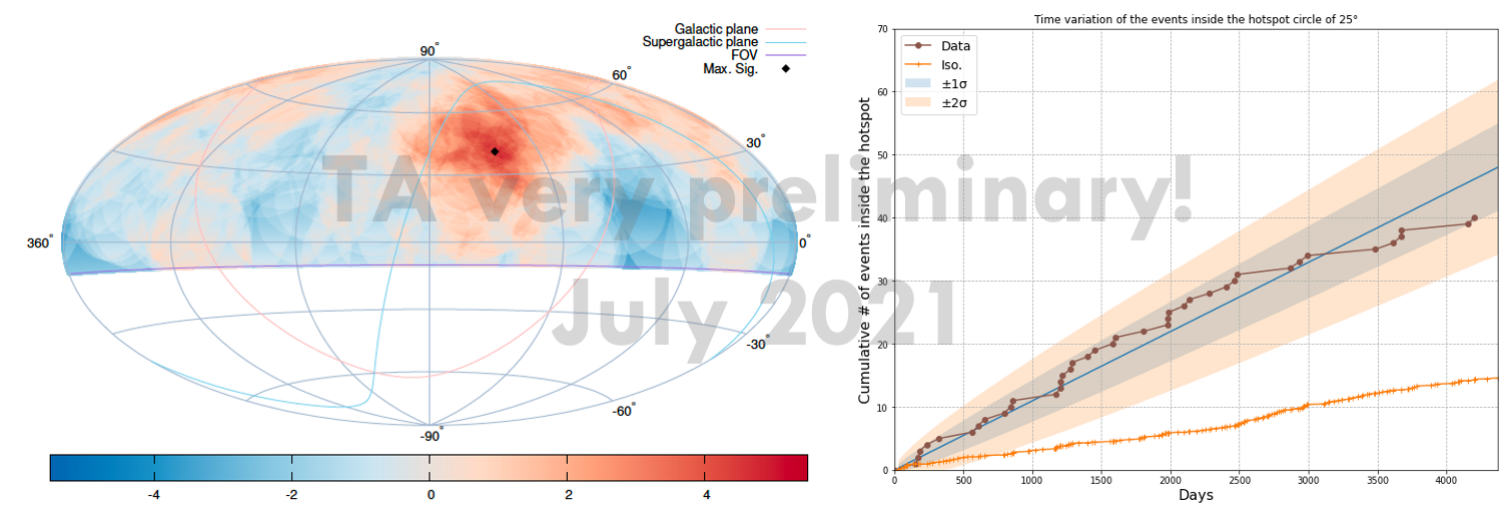

Figure 1: The sky map using Hammer projection and the increase rate of the events inside the hotspot circle. The left figure shows the hotspot sky map with the maximum Li-Ma significance position using the black dot. In the right figure, the brown dots represent the cumulative number of observed events inside the hotspot region, and the orange crosses indicate that of isotropic background events, respectively. The light blue and peach bands show $\pm 1 \sigma$ and $\pm 2 \sigma$ deviations from a linear increase rate.

calculated to be $5.1 \sigma$. The Li-Ma significance map is shown in the left of Fig. 1. The probability of detecting such clustered events by chance is estimated to be $6.8 \times 10^{-4}$, corresponding to $\sim 3.2 \sigma$, by MC simulations.

In addition, we conducted an independent analysis by dividing our data into two periods: first 5 -year and last 7-year. At the hotspot center, which is defined by the 12-year data, the Li-Ma significance is $5.0 \sigma$, the hotspot, for the first 5 -year dataset; however, it is calculated to be $2.3 \sigma$ for the last 7-year data, which is a "warm spot". Based on this, the time variation of the hotspot was investigated by counting the cumulative number of events inside the hotspot as time goes on, shown in the right of Fig. 1. Inside the hotspot circle defined by the 12-year dataset, the cumulative number of observed events and that of isotropic background events are shown with $\pm 1 \sigma$ and $\pm 2 \sigma$ bands deviations from a linear increase rate. We conclude that the increase rate of the events inside the hotspot circle is consistent with a constant within $\pm 1 \sigma$ fluctuation.

\section{New excess of events at slightly lower energy}

For the hotspot analysis, the dataset with energy greater than $57 \mathrm{EeV}$ was used. Now, we lower the energy threshold slightly. While searching for anomalies at $E \geq 10^{19.4} \mathrm{eV}, 10^{19.5} \mathrm{eV}$, and $10^{19.6} \mathrm{eV}$, we found new excesses of events in arrival direction distribution at all three energies. By adopting the same methodology used for the original hotspot study [8], we conducted the oversampling analysis with $20^{\circ}$ angular windows. The data selection criteria are tighter than those for the hotspot to keep the energy and angular resolution best because we search for the anisotropy in slightly lower energy. Note that the 11-year data was used for this analysis.

Fig. 2 shows the sky maps of the Li-Ma significance using a $20^{\circ}$-circle angular window for $10^{19.4} \mathrm{eV}, 10^{19.5} \mathrm{eV}, 10^{19.6} \mathrm{eV}$, and $57 \mathrm{EeV}$, respectively. The black dots represent the positions of the maximum Li-Ma significance, the maximum excess of events compared to the isotropy. For three lower energy thresholds, new excesses of events are continuously observed around the upper 

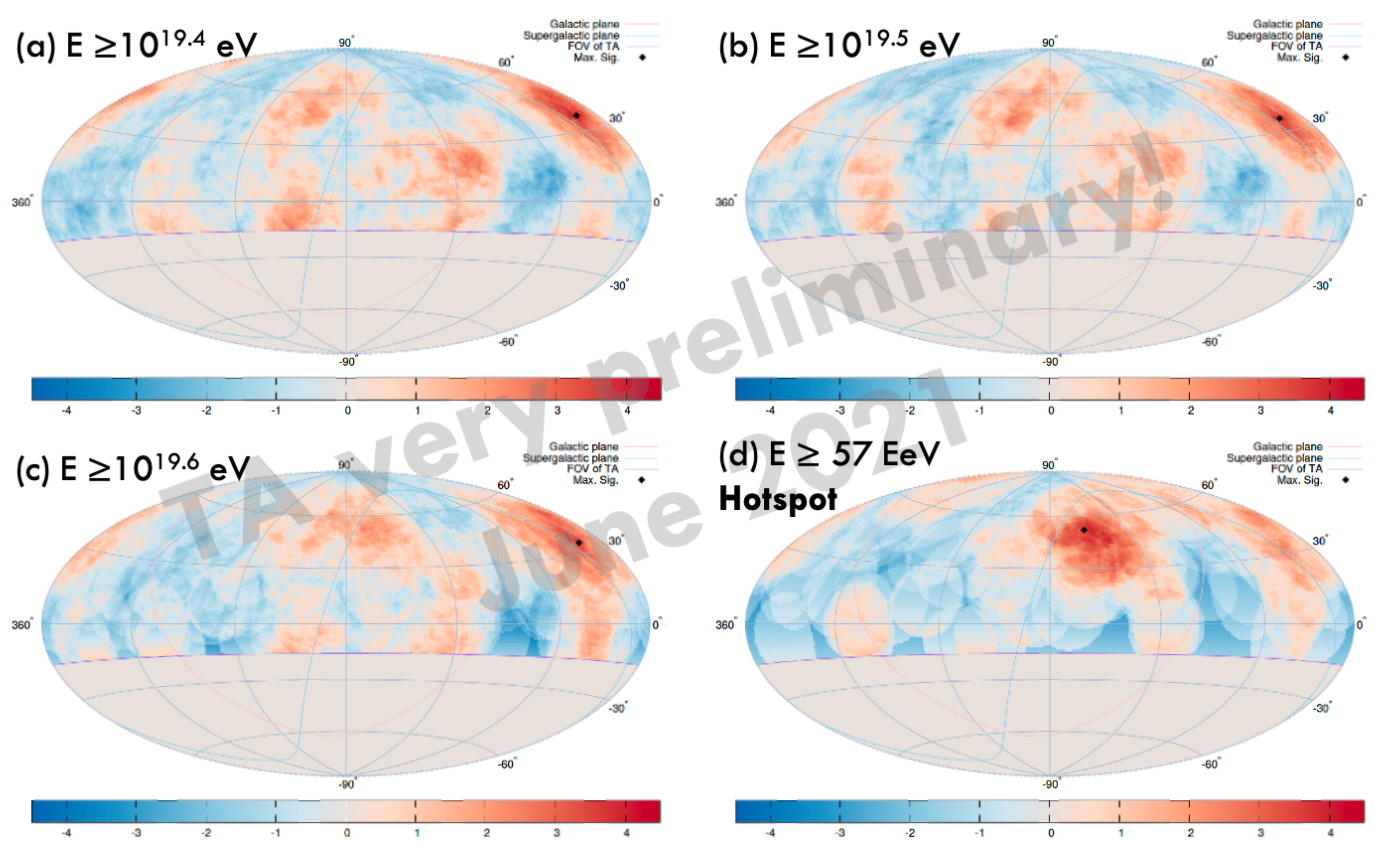

Figure 2: The sky maps using Hammer projections. It is shown that the Li-Ma significance using a $20^{\circ}$ circle angular window in the equatorial coordinates for different energy thresholds: (a) $E \geq 10^{19.4} \mathrm{eV}$, (b) $E \geq 10^{19.5} \mathrm{eV}$, (c) $E \geq 10^{19.6} \mathrm{eV}$, and (d) $E \geq 57 \mathrm{EeV}$. The color code indicates an excess (red) and a deficit (blue) of events compared to the isotropy at each grid point. The black dot represents the position of the maximum Li-Ma significance.

right side of the sky maps in Fig. 2. For the highest energy, which has the hotspot, the maximum significance position shifts from the new excesses in the lower energy to the center region in the figure, the hotspot position, but there is still a warm spot as others for lower energy events.

The new excesses of events in the arrival direction are summarized as follows: the Li-Ma significances are calculated to be $4.4 \sigma$ at $\left(17.4^{\circ}, 36.0^{\circ}\right), 4.2 \sigma$ at $\left(19.0^{\circ}, 35.1^{\circ}\right)$, and $4.0 \sigma$ at $\left(19.7^{\circ}, 34.6^{\circ}\right)$ for $E \geq 10^{19.4} \mathrm{eV}, E \geq 10^{19.5} \mathrm{eV}$, and $E \geq 10^{19.6} \mathrm{eV}$, respectively. Their positions are marked with the black dots in Fig. 2.

In addition, we checked the time variation of the excess by dividing the data into two periods as the same for the hotspot. The sky maps of the first 5-year data and the last 6-year data for $E \geq 10^{19.4} \mathrm{eV}$ are shown in Fig. 3. Unlike the hotspot, there is no apparent difference between the first 5- and last 6-year data. Both of them have similar local significances toward the new excess region, which are around $3 \sigma$. It indicates that steady excesses have been observed in this region.

To find out what causes this excess of events, we checked the large-scale structure of the universe. We found that the place where the new excess of events appears is in the region of the Perseus-Pisces supercluster (PPSC), which is one of the closest superclusters in the northern hemisphere. According to studies on the large scale structure of the universe [16-18], the PPSC is a part of the filaments of galaxies that extend to the Pegasus cluster. That elongated galaxy distribution is the most dominant, dense structure toward the excess region. There is no dense structure between us and the PPSC. Beyond the supercluster, at least up to $\sim 300 \mathrm{Mpc}$, no prominent 


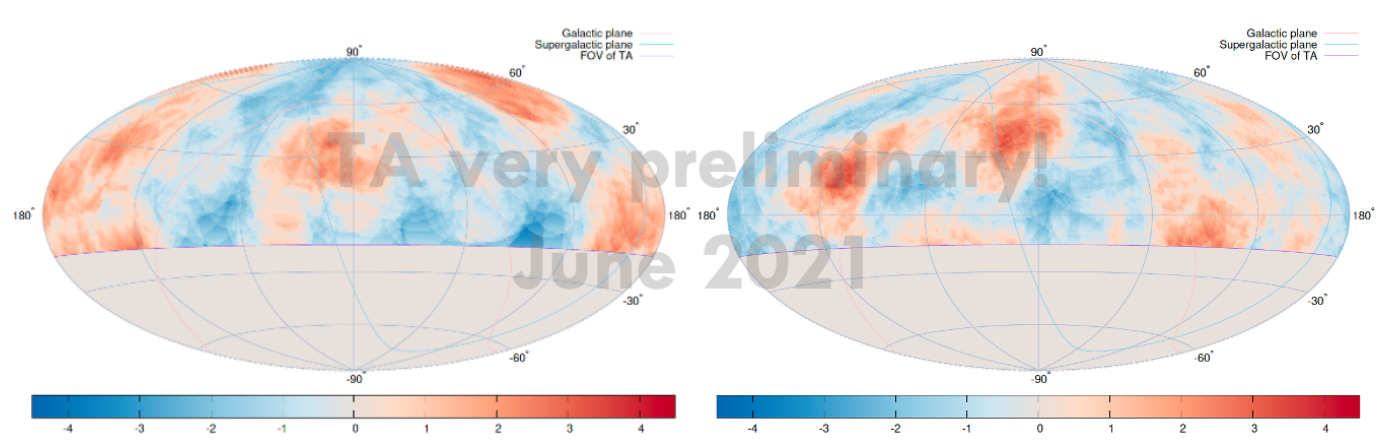

Figure 3: The sky maps using Hammer projections. For the first 5-year data (left) and the last 6-year data (right), the Li-Ma significances of the data with $E \geq 10^{19.4} \mathrm{eV}$ are shown in the equatorial coordinates. The color code is the same as Fig. 2, but the center of the sky map is rotated by $180^{\circ}$. As a result, it is $0^{\circ}$ in the right ascension at the center, which shows the new excess more clearly around the center of the sky map.

large-scale structure other than the PPSC. This implies that we need to pay attention that the PPSC could be responsible for the new excess of events.

Expanded sky maps are shown in Fig. 4 to check the distribution of the Perseus-Pisces supercluster and the new excess of events on the sky. The black asterisks are the positions of the representative members of the PPSC, which are clusters of galaxies and groups of galaxies. For each energy threshold, the excess region is coincident with the distribution of the PPSC very well overall. As we mentioned above, in the highest energy, $E \geq 57 \mathrm{EeV}$, we have a warm spot other than the hotspot, and that warm spot also overlaps with the PPSC. In the figure, the cyan diamonds represent the center position of the excess, where the maximum Li-Ma significance appears, and the green squares indicate the center of the PPSC, $\left(20.9^{\circ}, 27.9^{\circ}\right)$ in the equatorial coordinates. The angular distances between those two center positions are as close as $8.6^{\circ}$ for $E \geq 10^{19.4} \mathrm{eV}, 7.4^{\circ}$ for $E \geq 10^{19.5} \mathrm{eV}$, and $6.8^{\circ}$ for $E \geq 10^{19.6} \mathrm{eV}$.

To determine the probability of having an excess on top of the PPSC by chance, we performed MC studies in the following way:

1. Find the angle $\theta_{o b s}$, between the position of the maximum Li-Ma significance of the data and the center of the PPSC, at each energy.

2. Throw MC trials with the same statistics as the data and perform the Li-Ma analysis of each trial.

3. Count as successes those within angle $\theta_{o b s}$ of the PPSC with a higher significance than the data with the PPSC.

4. Calculate the probability of having an excess on top of the PPSC by chance.

We generated $5 \times 10^{5} \mathrm{MC}$ trials. For $E \geq 10^{19.4} \mathrm{eV}$, there are 49 instances out of $5 \times 10^{5} \mathrm{MC}$ trials having a greater Li-Ma significance than $4.4 \sigma$ within $8.6^{\circ}$ from the center of the PPSC, which corresponds to $3.7 \sigma$. In the same way, for $E \geq 10^{19.5} \mathrm{eV}$, there are 52 cases out of the given MC trials within $7.4^{\circ}$, whose Li-Ma significances greater than the pre-trial significance of $4.2 \sigma$, and its post-trial significance is estimated to be $3.7 \sigma$. For $E \geq 10^{19.6} \mathrm{eV}$, we have 134 realizations with greater Li-Ma significance than $4.0 \sigma$ within $6.8^{\circ}$ from the PPSC, corresponding to the post-trial significance of $3.5 \sigma$. MC trials having an excess of events on top of the PPSC are not common at all. This motivates us to conduct further statistical analyses, including a cross-correlation study 

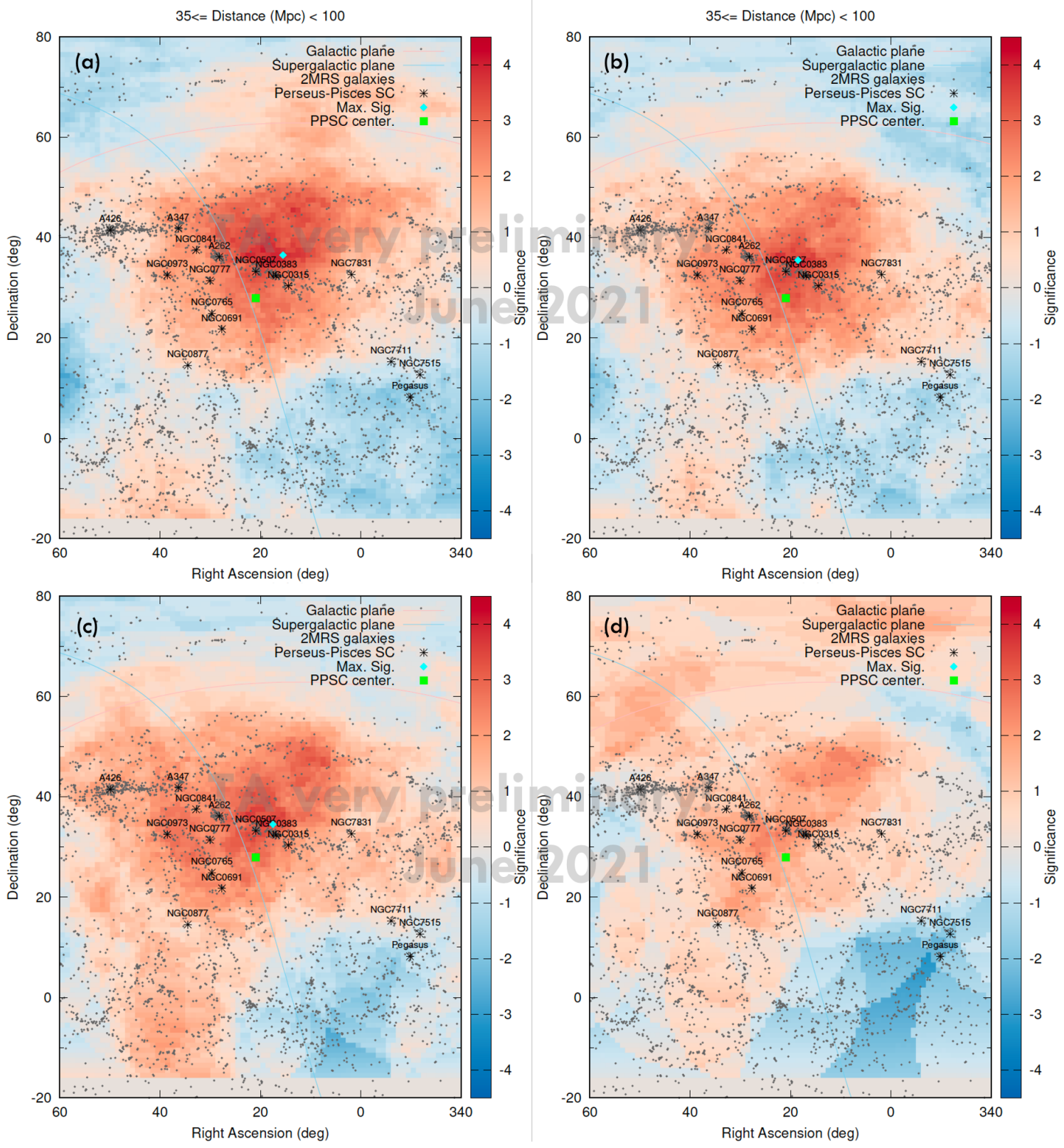

Figure 4: The expanded sky maps of the excesses of events with the nearby galaxies (gray dots) and the representative members of the Perseus-Pisces supercluster (asterisks) for (a) $E \geq 10^{19.4} \mathrm{eV}$, (b) $E \geq 10^{19.5} \mathrm{eV}$, (c) $E \geq 10^{19.6} \mathrm{eV}$, and (d) $E \geq 57 \mathrm{EeV}$, respectively. The cyan diamonds mark the positions having the maximum Li-Ma significances, and the green square represents the center of the Perseus-Pisces supercluster. The color code is the same as Fig. 2, but no projection is used. 
between the data and the members of the PPSC.

\section{Summary}

We have persistent hints of intermediate angular scale anisotropies, the hotspot, at the highest energies, $E>57 \mathrm{EeV}$, with its post-trial probability estimated to be $3.2 \sigma$. A new excess appears in slightly lower energy events with the local Li-Ma significance of $4.2 \sigma$. Behind the new excess, there is the Perseus-Pisces supercluster, which extends from the Perseus cluster to the Pegasus cluster. We performed MC studies to calculate the probability of having an excess on top of the Perseus-Pisces supercluster by chance. It is estimated to be $3.6 \sigma$, indicating a rare occurrence. More analyses, such as a cross-correlation analysis between the data and the members of the Perseus-Pisces supercluster, are underway and will be published elsewhere.

\section{References}

[1] R. U. Abbasi et al. [High Resolution Fly's Eye collab.], PRL 100 (2008) 101101.

[2] J. Abraham et al. [Pierre Auger collab.], PRL 101 (2008) 061101.

[3] T. Abu-Zayyad et al. [Telescope Array collab.], ApJL 768 (2013) L1.

[4] R. U. Abbasi et al. [High Resolution Fly's Eye collab.], PRL 104 (2010) 161101.

[5] A. Aab et al. [Pierre Auger collab.], PRD 90 (2014) 122006.

[6] R. U. Abbasi et al. [Telescope Array collab.], Astropart. Phys. 64 (2015) 49.

[7] R. U. Abbasi et al. [Telescope Array collab.], ApJ 858 (2018) 27.

[8] R. U. Abbasi et al. [Telescope Array collab.], ApJL 790 (2014) L21.

[9] A. Aab et al. [Pierre Auger collab.], ApJL 853 (2018) L29.

[10] K. Kawata et al. [Telescope Array collab.], PoS (ICRC2019) 310.

[11] T. Abu-Zayyad, et al. [Telescope Array collab.], NIM-A 689 (2012) 87.

[12] H. Tokuno et al. [Telescope Array collab.], NIM-A 676 (2012) 54.

[13] T. Abu-Zayyad, et al. [Telescope Array collab.], ApJL 768 (2013) L1.

[14] R. U. Abbasi et al. [Telescope Array collab.], ApJ 862 (2018) 91.

[15] T. P. Li and Y. Q. Ma, ApJL 272 (1983) 317

[16] D. J. Batuski and J. O. Burns, ApJ 299 (1984) 5.

[17] J. P. Huchra et al., ApJS 199 (2012) 22.

[18] H. M. Courtois et al., AJ 146 (2013) 69. 


\section{Full Authors List: Telescope Array Collaboration}

R.U. Abbasi ${ }^{1,2}$, T. Abu-Zayyad ${ }^{1,2}$, M. Allen ${ }^{2}$, Y. Arai $^{3}$, R. Arimura ${ }^{3}$, E. Barcikowski², J.W. Belz ${ }^{2}$, D.R. Bergman ${ }^{2}$, S.A. Blake ${ }^{2}$, I. Buckland ${ }^{2}$, R. Cady $^{2}$, B.G. Cheon ${ }^{4}$, J. Chiba ${ }^{5}$, M. Chikawa ${ }^{6}$, T. Fujii ${ }^{7}$, K. Fujisue ${ }^{6}$, K. Fujita ${ }^{3}$, R. Fujiwara ${ }^{3}$, M. Fukushima ${ }^{6}$, R. Fukushima ${ }^{3}$, G. Furlich ${ }^{2}$, R. Gonzalez ${ }^{2}$, W. Hanlon ${ }^{2}$, M. Hayashi ${ }^{8}$, N. Hayashida ${ }^{9}$, K. Hibino ${ }^{9}$, R. Higuchi ${ }^{6}$, K. Honda ${ }^{10}$, D. Ikeda ${ }^{9}$, T. Inadomi ${ }^{11}$, N. Inoue ${ }^{12}$, T. Ishii ${ }^{10}$, H. Ito ${ }^{13}$, D. Ivanov ${ }^{2}$, H. Iwakura ${ }^{11}$, A. Iwasaki ${ }^{3}$, H.M. Jeong ${ }^{14}$, S. Jeong ${ }^{14}$, C.C.H. Jui ${ }^{2}$, K. Kadota ${ }^{15}$, F. Kakimoto ${ }^{9}$, O. Kalashev ${ }^{16}$, K. Kasahara ${ }^{17}$, S. Kasami ${ }^{18}$, H. Kawai ${ }^{19}$, S. Kawakami ${ }^{3}$, S. Kawana ${ }^{12}$, K. Kawata ${ }^{6}$, I. Kharuk ${ }^{16}$, E. Kido ${ }^{13}$, H.B. Kim ${ }^{4}$, J.H. Kim ${ }^{2}$, J.H. Kim ${ }^{2}$, M.H. Kim ${ }^{14}$, S.W. Kim ${ }^{14}$, Y. Kimura ${ }^{3}$, S. Kishigami ${ }^{3}$, Y. Kubota ${ }^{11}$, S. Kurisu ${ }^{11}$, V. Kuzmin ${ }^{16,36}$, M. Kuznetsov ${ }^{16,20}$, Y.J. Kwon ${ }^{21}$, K.H. Lee ${ }^{14}$, B. Lubsandorzhiev ${ }^{16}$, J.P. Lundquist ${ }^{2,22}$, K. Machida ${ }^{10}$, H. Matsumiya ${ }^{3}$, T. Matsuyama ${ }^{3}$, J.N. Matthews ${ }^{2}$, R. Mayta ${ }^{3}$, M. Minamino ${ }^{3}$, K. Mukai ${ }^{10}$, I. Myers ${ }^{2}$, S. Nagataki ${ }^{13}$, K. Nakai ${ }^{3}$, R. Nakamura ${ }^{11}$, T. Nakamura ${ }^{23}$, T. Nakamura ${ }^{11}$, Y. Nakamura ${ }^{11}$, A. Nakazawa ${ }^{11}$, E. Nishio ${ }^{18}$, T. Nonaka ${ }^{6}$, H. Oda ${ }^{3}$, S. Ogio ${ }^{3,24}$, M. Ohnishi ${ }^{6}$, H. Ohoka ${ }^{6}$, Y. Oku ${ }^{18}$, T. Okuda ${ }^{25}$, Y. Omura ${ }^{3}$, M. Ono ${ }^{13}$, R. Onogi ${ }^{3}$, A. Oshima ${ }^{3}$, S. Ozawa ${ }^{26}$, I.H. Park ${ }^{14}$, M. Potts ${ }^{2}$, M.S. Pshirkov ${ }^{16,27}$, J. Remington ${ }^{2}$, D.C. Rodriguez ${ }^{2}$, G.I. Rubtsov ${ }^{16}$, D. Ryu ${ }^{28}$, H. Sagawa ${ }^{6}$, R. Sahara ${ }^{3}$, Y. Saito ${ }^{11}$, N. Sakaki ${ }^{6}$, T. Sako ${ }^{6}$, N. Sakurai ${ }^{3}$, K. Sano ${ }^{11}$, K. Sato ${ }^{3}$, T. Seki ${ }^{11}$, K. Sekino ${ }^{6}$, P.D. Shah ${ }^{2}$, Y. Shibasaki ${ }^{11}$, F. Shibata ${ }^{10}$, N. Shibata ${ }^{18}$, T. Shibata ${ }^{6}$, H. Shimodaira ${ }^{6}$, B.K. Shin ${ }^{28}$, H.S. Shin ${ }^{6}$, D. Shinto ${ }^{18}$, J.D. Smith ${ }^{2}$, P. Sokolsky ${ }^{2}$, N. Sone ${ }^{11}$, B.T. Stokes ${ }^{2}$, T.A. Stroman ${ }^{2}$, Y. Takagi ${ }^{3}$,

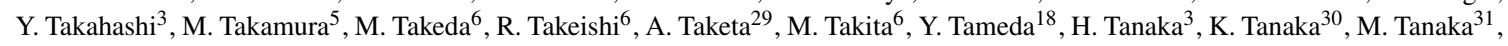
Y. Tanoue ${ }^{3}$, S.B. Thomas ${ }^{2}$, G.B. Thomson ${ }^{2}$, P. Tinyakov ${ }^{16,20}$, I. Tkachev ${ }^{16}$, H. Tokuno ${ }^{32}$, T. Tomida ${ }^{11}$, S. Troitsky ${ }^{16}$, R. Tsuda ${ }^{3}$, Y. Tsunesada ${ }^{3,24}$, Y. Uchihori ${ }^{33}$, S. Udo ${ }^{9}$, T. Uehama ${ }^{11}$, F. Urban ${ }^{34}$, T. Wong ${ }^{2}$, K. Yada ${ }^{6}$, M. Yamamoto ${ }^{11}$, K. Yamazaki ${ }^{9}$, J. Yang ${ }^{35}$, K. Yashiro ${ }^{5}$, F. Yoshida ${ }^{18}$, Y. Yoshioka ${ }^{11}$, Y. Zhezher ${ }^{6,16}$, and Z. Zundel ${ }^{2}$

${ }^{1}$ Department of Physics, Loyola University Chicago, Chicago, Illinois, USA

${ }^{2}$ High Energy Astrophysics Institute and Department of Physics and Astronomy, University of Utah, Salt Lake City, Utah, USA

${ }^{3}$ Graduate School of Science, Osaka City University, Osaka, Osaka, Japan

${ }^{4}$ Department of Physics and The Research Institute of Natural Science, Hanyang University, Seongdong-gu, Seoul, Korea

${ }^{5}$ Department of Physics, Tokyo University of Science, Noda, Chiba, Japan

${ }^{6}$ Institute for Cosmic Ray Research, University of Tokyo, Kashiwa, Chiba, Japan

7 The Hakubi Center for Advanced Research and Graduate School of Science, Kyoto University, KitashirakawaOiwakecho, Sakyo-ku, Kyoto, Japan

${ }^{8}$ Information Engineering Graduate School of Science and Technology, Shinshu University, Nagano, Nagano, Japan

${ }^{9}$ Faculty of Engineering, Kanagawa University, Yokohama, Kanagawa, Japan

${ }_{10}^{10}$ Interdisciplinary Graduate School of Medicine and Engineering, University of Yamanashi, Kofu, Yamanashi, Japan

${ }^{11}$ Academic Assembly School of Science and Technology Institute of Engineering, Shinshu University, Nagano, Nagano, Japan

12 The Graduate School of Science and Engineering, Saitama University, Saitama, Saitama, Japan

13 Astrophysical Big Bang Laboratory, RIKEN, Wako, Saitama, Japan

${ }_{15}^{14}$ Department of Physics, SungKyunKwan University, Jang-an-gu, Suwon, Korea

15 Department of Physics, Tokyo City University, Setagaya-ku, Tokyo, Japan

16 Institute for Nuclear Research of the Russian Academy of Sciences, Moscow, Russia

17 Faculty of Systems Engineering and Science, Shibaura Institute of Technology, Minato-ku, Tokyo, Japan

18 Department of Engineering Science, Faculty of Engineering, Osaka Electro-Communication University, Neyagawashi, Osaka, Japan

${ }^{19}$ Department of Physics, Chiba University, Chiba, Chiba, Japan

${ }^{20}$ Service de Physique Théorique, Université Libre de Bruxelles, Brussels, Belgium

${ }^{21}$ Department of Physics, Yonsei University, Seodaemun-gu, Seoul, Korea

22 Center for Astrophysics and Cosmology, University of Nova Gorica, Nova Gorica, Slovenia

23 Faculty of Science, Kochi University, Kochi, Kochi, Japan

${ }_{24}$ Nambu Yoichiro Institute of Theoretical and Experimental Physics, Osaka City University, Osaka, Osaka, Japan

25 Department of Physical Sciences, Ritsumeikan University, Kusatsu, Shiga, Japan

${ }^{26}$ Quantum ICT Advanced Development Center, National Institute for Information and Communications Technology, Koganei, Tokyo, Japan

27 Sternberg Astronomical Institute, Moscow M.V. Lomonosov State University, Moscow, Russia

${ }^{28}$ Department of Physics, School of Natural Sciences, Ulsan National Institute of Science and Technology, UNIST-gil, Ulsan, Korea

${ }_{29}$ Earthquake Research Institute, University of Tokyo, Bunkyo-ku, Tokyo, Japan

${ }^{30}$ Graduate School of Information Sciences, Hiroshima City University, Hiroshima, Hiroshima, Japan

${ }^{31}$ Institute of Particle and Nuclear Studies, KEK, Tsukuba, Ibaraki, Japan

${ }^{32}$ Graduate School of Science and Engineering, Tokyo Institute of Technology, Meguro, Tokyo, Japan

33 Department of Research Planning and Promotion, Quantum Medical Science Directorate, National Institutes for Quantum and Radiological Science and Technology, Chiba, Chiba, Japan 
${ }^{34}$ CEICO, Institute of Physics, Czech Academy of Sciences, Prague, Czech Republic

${ }^{35}$ Department of Physics and Institute for the Early Universe, Ewha Womans University, Seodaaemun-gu, Seoul, Korea

\section{Acknowledgements}

The Telescope Array experiment is supported by the Japan Society for the Promotion of Science(JSPS) through Grants-in-Aid for Priority Area 431, for Specially Promoted Research JP21000002, for Scientific Research (S) JP19104006, for Specially Promoted Research JP15H05693, for Scientific Research (S) JP15H05741 and JP19H05607, for Science Research (A) JP18H03705, for Young Scientists (A) JPH26707011, and for Fostering Joint International Research (B) JP19KK0074, by the joint research program of the Institute for Cosmic Ray Research (ICRR), The University of Tokyo; by the Pioneering Program of RIKEN for the Evolution of Matter in the Universe (r-EMU); by the U.S. National Science Foundation awards PHY-1404495, PHY-1404502, PHY-1607727, PHY-1712517, PHY-1806797 and PHY-2012934; by the National Research Foundation of Korea (2017K1A4A3015188, 2020R1A2C1008230, \& 2020R1A2C2102800) ; by the Ministry of Science and Higher Education of the Russian Federation under the contract 075-15-2020778, RFBR grant 20-02-00625a (INR), IISN project No. 4.4501.18, and Belgian Science Policy under IUAP VII/37 (ULB). This work was partially supported by the grants ofThe joint research program of the Institute for Space-Earth Environmental Research, Nagoya University and Inter-University Research Program of the Institute for Cosmic Ray Research of University of Tokyo. The foundations of Dr. Ezekiel R. and Edna Wattis Dumke, Willard L. Eccles, and George S. and Dolores Doré Eccles all helped with generous donations. The State of Utah supported the project through its Economic Development Board, and the University of Utah through the Office of the Vice President for Research. The experimental site became available through the cooperation of the Utah School and Institutional Trust Lands Administration (SITLA), U.S. Bureau of Land Management (BLM), and the U.S. Air Force. We appreciate the assistance of the State of Utah and Fillmore offices of the BLM in crafting the Plan of Development for the site. Patrick A. Shea assisted the collaboration with valuable advice and supported the collaboration's efforts. The people and the officials of Millard County, Utah have been a source of steadfast and warm support for our work which we greatly appreciate. We are indebted to the Millard County Road Department for their efforts to maintain and clear the roads which get us to our sites. We gratefully acknowledge the contribution from the technical staffs of our home institutions. An allocation of computer time from the Center for High Performance Computing at the University of Utah is gratefully acknowledged.

36 Deceased 